\title{
PLAN DE CAPACITACION DE INGLES PARA PROFESORES DE PRIMARIA ZONA URBANA DEL MUNICIPIO DE NEIVA-NIVEL INTRODUCTORIO I - II
}

\section{INTRODUCCION}

Por: Luis Evelio Vanegas Rubio Profesor Titular Lenguas Modernas Universidad Surcolombiana.

Lilian Cecilia Zambrano Castillo Profesora tiempo completo Lenguas Modernas

Universidad Surcolombiana

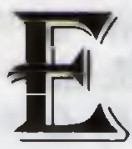

ste plan de resultado de la investigación titulada "LA ENSEÑANZA DEL IDIOMA INGLES EN LA EDUCACIÓN BÁSICA PRIMARIA EN EL MUNICIPIO DE NEIVA". Participaron en el diagnóstico en forma directa 101 centros educativos (7) oficiales y 30 no oficiales de la zona urbana representados por sus rectores y 257 profesores (190 de establecimientos oficiales y 68 de los no oficiales). Así mismo participaron los 8 jefes de núcleo que hay en el municipio de Neiva zona urbana. El aspecto académico fue estructurado por los profesores Luis Evelio vanegas Rubio y Lilian Cecilia Zambrano Castillo con la colaboración de otros profesores del Departamento de Lenguas Modernas de la Universidad Surcolombiana. Se contó con el recurso bibliográfico de la Universidad, el cual en esta área está muy actualizado y el propio de cada uno de los investigadores.

Toda esta estructura nos permite presentar un plan de capacitación en el cual la alta participación y motivación de sus participantes y la calidad académica de sus contenidos hacen prever éxitos en esta área del conocimiento. En esta primera etapa se propone trabajar con 360 profesores cada uno de los cuales participará en 270 horas repartidas en dos cursos. El contenido de cada curso girará en tomo a :

a) Adquisición o dominio de la lengua inglesa

b) Conocimiento del desarrollo socioafectivo lingüístico del niño

c) Análisis de los contenidos de Inglés de los grados tercero, cuarto y quinto de primaria

d) Metodología de la enseñanza de esos grados

e) Su sistema de evaluación

Nuestra propuesta contempla el inicio de la enseñanza y de la preparación de los profesores en el grado tercero debido a conceptos teóricos y a coyunturas prácticas de nuestro contexto. Entre ellas tenemos:

Porque el estado de desarrollo lingüístico del niño de grado tercero 
facilita la acción docente e incluso algunas investigaciones sugieren que es la etapa ideal del aprendizaje de una lengua extranjera.

$\checkmark$ Porque hacia la edad del grado tercero el niño presenta un desarrollo de sus valores culturales mas claros, lo cual le permite identificar los valores de la otra cultura sin conflictos con la suya.

$\checkmark$ Porque ante la inmensa cantidad de docentes que hay que capacitar, bajo esta propuesta, se dividirá en dos grandes etapas lo cual hace al proyecto operativo y financieramente más viable.

Este plan de capacitación fue presentado a la Comisión de Empalme del Gobierno Departamental en diciembre de 1994. En febrero de 1995 se le presento al señor Gobernador Jaime Losada Perdomo con otros proyectos de la Facultad de Educación de la Universidad Surcolombiana. El señor Gobernador apoyo este proyecto y lo remitió a estudio y análisis en el Consejo Regional de Política Económica y Social- CORPES CENTRO ORIENTE, el cual lo aprobó y le asignó una partida de veintiseis millones de pesos $(\$ 26.000 .000,00)$ para capacitar a 240 profesores de primaria del municipio de Neiva. Estamos en espera que se protocolice el convenio respectivo entre la Gobernación, El CORPES , la Secretaria de Educación y la Universidad Surcolombiana para dar comienzo a este importante y necesario proyecto. Esperamos que su inicio sea en el mes de noviembre de 1995.
La necesidad de comunicación y el desarrollo tecnológico han roto las distancias entre los diferentes pueblos del mundo, creando a su vez la necesidad recíproca de aprendizaje de sus lenguas para una mayor comprensión e intercambio de intereses ya sean estos de carácter comercial. financiero, cultural, recreativo, etc

La Ley General de Educación al extender la enseñanza obligatoria de un idioma extranjero a todos los grados de la educación básica, visualizo esta situación e interpretó la realidad colombiana como la de un estado democrático y pluralista con relaciones internacionales abiertas a todos los países, y por ende su necesidad de fomentar el aprendizaje de lenguas extranjeras que faciliten la comunicación con el resto del mundo.

Además es innegable la preponderancia del idioma Inglés en las comunicaciones internacionales especialmente a nivel de desarrollo tecnológico y cultural. Colombia se enmarca dentro del conglomerado de países en pleno desarrollo tecnológico y necesita dotar a sus profesionales del dominio de este idioma para permitir una comunicación mas eficaz y lograr mejores resultados de sus relaciones internacionales.

Por otra parte, la gran mayoría de las instituciones educativas en Colombia, oficiales y privadas, en la actualidad enseñan Inglés en los grados sexto a 
noveno y algunos continúan en los grados décimo y undécimo. El Departamento del Huila no es excepción y ha creado una pequeña estructura en esta área del conocimiento que debe ser aprovechada para extenderla a la educación básica primaria y en esta dar cumplimiento a la Ley General de Educación.

Pero esta enseñanza debe darse bajo una rigurosa planeación académica para evitar efectos negativos que le creen al niño la imposibilidad de acceder al conocimiento del idioma por la elevación del "filtro afectivo" concepto bien conocido en los ámbitos de los lingüistas de hoy en día.

Desafortunadamente, en la actualidad. la mayoría de los profesores de primaria carecen de un manejo básico del idioma Inglés y de las estrategias metodológicas para enseñarlo. Esto esta demostrado en las visitas, reuniones, entrevistas $y$ encuestas aplicadas a 190 profesores en 71 establecimientos educativos oficiales de educación básica primaria del municipio de Neiva y a 68 profesores del sector no oficial.

Se deduce entonces la inminente necesidad de dar inicio a estos cursos de capacitación para proporcionar al docente las herramientas básicas necesarias para poder enfrentar el gran reto de enseñar Inglés en los grados de educación básica primaria y dar cumplimiento a la nueva Ley 115 de 1994.

\section{OBJETIVOS}

\section{GENERALES}

1. Contribuir con el desarrollo educativo del municipio de Neiva y el Departamento del Huila en el área especifica de la enseñanza aprendizaje del Inglés.

2. Facilitar la implementación de la Ley General de Educación en el área especifica de Inglés en la educación básica primaria en Neiva y el Departamento del Huila.

\section{ESPECIFICOS}

1. Introducir al profesor en el manejo básico del idioma Inglés.

2. Dar a conocer las características del estado de desarrollo socio-afectivo y lingüístico del niño.

3. Analizar los contenidos de la enseñanza del Ingles en los grados tercero, cuarto y quinto de primaria.

4. Presentar diferentes teorías y enfoques metodológicos sobre la enseñanza de Inglés para niños.

5. Señalar diferentes sistemas de evaluación acordes con lo establecido en el capitulo VI del Decreto 1860 de 1994.

\section{ESTRATEGIA}

La primera etapa del "Plan de Capacitación de Inglés para profesores de primaria zona urbana del Municipio de 
Neiva Nivel Introductorio I y II comprende cinco bloques temáticos a saber :

1. Adquisición de la lengua Inglesa.

2. Conocimiento del estado de desarrollo socio-afectivo-lingüístico del niño

3. Análisis de contenido de la enseñanza del Inglés en los grados tercero, cuarto y quinto.

4. Metodología de la enseñanza del Inglés en esos grados.

5. Sistemas de evaluación.

\section{BLOQUE TEMATICO No. 1} ADQUISICION DE LA

\section{LENGUA INGLESA}

La mayoría de los profesores de primaria, según el diagnóstico realizado, solamente presentan los estudios de Inglés de bachillerato que, además califican de no ser buenos. Por eso, con este bloque temático se busca el mejoramiento del conocimiento y uso del idioma Inglés por parte del profesor.- Se enfoca en el idioma como medio de comunicación, integrando las cuatro babilidades lingüísticas-escucharhablar-leer y escribir y en el estudio de las necesidades de las estructuras gramaticales y el vocabulario.

El contenido de este bloque temático está basado en las funciones del lenguaje. La selección de estas funciones se hizo desde el punto de vista del "Enfoque Comunicativo" y teniendo en cuenta al mismo tiempo, los contenidos de Inglés del grado tercero de primaria. Es importante resaltar aqui, que el diseño de este plan de capacitación permite la teoría-práctica, en cuanto que el profesor de primaria va mejorando sus conocimientos de Inglés sobre funciones específicos y al mismo tiempo las va aplicando en la enseñanza en sus grados respectivos en su' establecimiento educativo.

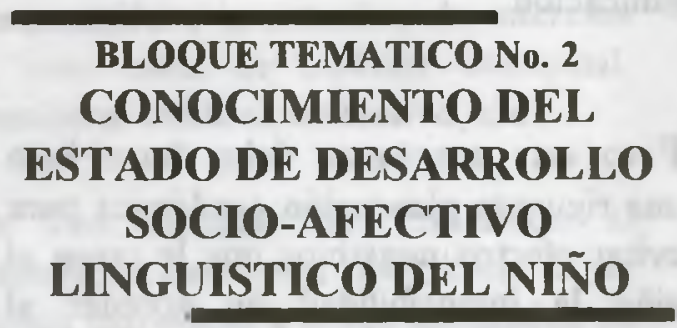

E1 conocimiento de este tema se considera como el de mayor importancia para un buen proceso de enseñanzaaprendizaje de una lengua extranjera en la edad de educación primaria. Estos factores de socialización, afectiva y estado de desarrollo de la lengua materna del niño van a determinar el qué, el cómo y el cuándo de la acción docente.

Es importante tener en cuenta que el aprendizaje de una segunda lengua no está aislado del crecimiento mental del niño. Por lo tanto, si se le pide que realice actividades para los cuales él aún no está listo, todo el proceso puede ser un fracaso y conlleva a que el niño pierda totalmente el interés y entusiasmo que generalmente lo caracterizan.

Todos los niños pasan por las mismas etapas de desarrollo pero los límites de estas etapas no son exactos, esto 
significa que el profesor debe estar alerta a los posibles cambios que se presentan en el niño $y$ fomentar actividades que se ajusten a su desarrollo normal.

Respecto al factor de socialización, cabe anotar que el niño entre los 6 y 12 años rápidamente desarrolla su conciencia social, y que su interés principal radica en las actividades de grupo: razón por la cual el profesor debe promover actividades comunicativas que le permitan al niño interactuar y participar activamente en el proceso de aprendizaje.

Otro factor muy importante es el desarrollo emocional o "Dominio afectivo", el cual está presente en el proceso de aprendizaje de una lengua extranjera. El profesor debe crear un ambiente de confianza en donde el niño tenga la oportunidad de expresar sus ideas y sentimientos, y participar libremente en las diversas tareas que conlleven el aprendizaje de la lengua extranjera.

Finalmente, el profesor debe tener en cuenta que el niño trae consigo ciertas habilidades lingüísticas que pueden ser explotadas en el desarrollo de la clase de una lengua extranjera. El niño tiene gran habilidad para captar el significado de los mensajes sin necesidad de entender cada palabra. Además tiene una gran capacidad de interactuar y hablar, la cual puede ser utilizada involucrando al niño en actividades como juegos, canciones, diálogos, etc. Este bloque temático se encargará de trabajar la teoría y la práctica de los factores aquí mencionados.

BLOQUE TEMATICO No. 3

ANALISIS DE LOS

CONTENIDOS DE LA

ENSEÑANZA DEL INGLES

EN LOS GRADOS 30., 40. Y 50.

El que enseñar en los grados 3o., 4o. y 5o. está determinado por los principios teóricos planteados en el enfoque que sustenta esta propuesta metodológica. La presentación del contenido está dividido en bloques que se componen de una o más funciones afectivas o lingüísticas en las cuales tratamos de delimitar en algo el vocabulario. las expresiones y algunas estructuras gramaticales (estas estructuras gramaticales son para conocimiento del profesor en el momento de planear sus clases; al estudiante no deberá hacérsele consciente de la estructura sino únicamente de su uso). A partir de estos bloques temáticos y con estas limitaciones, la creatividad del profesor y el contexto específico en que se desarrolle el acto educativo determinarán los mayores o menores avances que se puedan lograr en tiempos determinados. De todas maneras vale la pena resaltar aquí que en esta propuesta metodológica es mucho mas importante la parte emocional y afectiva del estudiante que la cantidad de material lingüístico que se logre dasarrollar Además, el material lingüístico que se 
propone en cada bloque temático no es para ser desarrollado en una sola clase, o que debe desarrollarse todo antes de ir al bloque siguiente. No. Lo que queremos es dar unas pautas mínimas necesarias para que el profesor haga sus propias combinaciones de elementos $y$ los desarrolle en el momento en que los considere apropiados.

Los contenidos y logros aquí planteados se analizarán y compararán con los que establezca el Ministerio de Educación Nacional para el grado 30 . de acuerdo con la Ley $115 / 94$.

\section{BLOQUE TEMATICO No. 4 METODOLOGIA DE LA ENSEÑANZA DEL INGLES EN LOS GRADOS 30., 40. Y 50.}

El profesor estará expuesto a varios criterios metodológicos y a la práctica de muchas actividades que conllevan esos conceptos. La selección, preparación y uso de materiales se trabajará en armonía de teoría y práctica que se realizará en los talleres del curso de capacitación y que luego el profesor aplicará en su establecimiento educativo. Los criterios metodológicos están fundamentados primordialmente en los planteamientos de James Asher en su libro "Learning Another Languaje Through Actions", de Stephen Crashen y Tracy Terrel en su libro Natural Approach; Susan Halliwell-Teaching English in the Primary Classroom y Scott \& Ytreberg- Teaching English to Children, así como los principios del Enfoque Comunicativo.
Algunos principios básicos que se establecen en el Método de Respuesta Física Total tiene que ver con el uso de órdenes o "commands" que se dan al niño, quien a su vez demuestra la comprensión del mensaje a través de una respuesta física. Su autor, James Sher. afirma que el niño al aprender su lengua materna pasa por una etapa similar, y que por medio de este contexto el niño va a adquirir una gran cantidad de vocabulario y estructuras gramaticales.

Krashen y Terrel en El Enfoque Natural señalan que el niño adquiere una segunda lengua siguiendo un proceso similar a la adquisición de su lengua materna. Por lo tanto, ellos establecen que la primera habilidad que se debe desarrollar es la habilidad de escucha: que la habilidad del habla surgirá espontáneamente, cuando el niño se sienta listo para hacerlo. En este enfoque se hace también mucho énfasis en la adquisición de vocabulario, pues sin un conocimiento básico de este componente lingüístico, el niño no será capaz de entender ni producir ningún mensaje.

Scott e Ytreberg por su parte, consideran que el proceso de adquisición de una lengua extranjera está determinado por las características que presente el niño en sus diversas etapas de desarrollo. Entre estas características se señala que el niño aún hasta la edad de 11 años no es consciente de las reglas gramaticales de su lengua nativa; esto implica que no será capaz de entender las reglas gramaticales de la lengua 
extranjera. Por esta razón, el profesor debe promover actividades comunicativas a través de las cuales la enseñanza de la gramática sea implícita y en donde ésta se convierte en un medio para llegar a un fin el cual es la comunicación.

\section{BLOQUE TEMATICO No. 5 SISTEMAS DE EVALUACION}

El enfoque de los diferentes enfoques de evaluación y testing del proceso de enseñanza-aprendizaje de una lengua extranjera, estarán en su conjunto diseñados para dar cabal cumplimiento a los criterios de evaluación continua, integral y cualitativa expresada en el Decreto 1860 de 1994, reglamentario de la Ley 115 del mismo año. La relación entre teorías y métodos de la enseñanzaaprendizaje de idiomas extranjeros y el sistema de evaluación, será íntima y deberá corresponder a los enfoques más modernos sobre este particular.

Bajo estos principios la evaluación será continua integral, cualitativa, y los informes a los estudiantes y a los padres de familia serán descriptivos y expresarán en forma sencilla los logros alcanzados por los estudiantes de acuerdo con los "criterios" o "indicadores" planteados de antemano. Esta valoración se dará en términos de EXCELENTE, BIEN O INSUFICIENTE.

\section{PLAN OPERATIVO}

Surge de la información obtenida de la investigación citada anteriormente de la cual extrajimos los siguiente aspectos relevantes:

$\checkmark$ En los 72 centros educativos oficiales funcionan 114 jornadas en cada una de las cuales funciona, en términos generales, un grupo de grado 30 ., uno de 4o., y uno de grado 50.

$\checkmark$ De los 190 profesores de establecimientos oficiales encuestados la gran mayoría prefiere tomar la capacitación los días sábados. Otros dos grandes grupos la prefieren en la noche de lunes a viernes y durante el tiempo de vacaciones de mitad de año

$\checkmark$ La gran mayoria de los profesores encuestados solicitaron la capacitación de Inglés a un NIVEL BÁSICO y de METODOLOGíA del mismo.

Obrando en concordancia con estas aspiraciones de los docentes y de los principios que rigen la enseñanza de idiomas extranjeros, proponemos que el Plan Operativo en esta primera etapa comprenda la capacitación en el Nivel Introductorio I y II de 360 profesores así:

\section{Grupos de 30 profesores}

Se trabajarán con 12 grupos de profesores en el primer semestre del año en el Nivel Introductorio No. 1. En el segundo semestre se trabajarán 
con esos mismos grupos el Nivel Introductorio No. 2.

$\checkmark$ Cada nivel se compone de 135 horạsclase y asesoría y seguimiento en el Centro Docente respectivo por parte del profesor-orientador quien lo visitará al menos una vez al mes.

$\checkmark$ Se trabajará con 5 grupos simultáneos durante 17 sábados del primer semestre del año con una intensidad de 8 horas cada sábado. Lo mismo se hará en el segundo semestre con el Nivel Introductorio No. 2.

$\checkmark$ Se trabajará con 4 grupos simultáneos en horario nocturno de lunes a viernes con una intensidad de 3 horas diarias durante el primer semestre. Esos mismos grupos recibirán en igual forma el Nivel Introductorio No.2 en el segundo semestre.

$\checkmark$ Se trabajará con 3 grupos simultáneos en las vacaciones de mitad de año. El Nivel Introductorio No.2 se les desarrollará en las vacaciones de fin de año.

En resumen cada grupo de profesores recibirá el Nivel Introductorio I en el primer semestre y el Nivel Introductorio II en el segundo semestre en los calendarios propuestos por ellos mismos de acuerdo con sus necesidades particulares.

\section{RECOMENDACIONES PARA LA SELECCION DE LOS PROFESORES PARA EL NIVEL INTRODUCTORIO I Y II}

Estas recomendaciones sólo pretenden enriquecer la lista de criterios que cada establecimiento o cada Núcleo Educativo o el CEP-Huila trace para la selección de los profesores que tomarán los cursos de Inglés del Nivel Introductorio I y II. Son criterios estrictamente académicos basados en teorias de aprendizaje y en la experiencia propia de los autores de este proyecto.

- Edad

- Experiencia en la enseñanza de Inglés

- Motivación hacia el idioma Inglés

- Mayor estudio de este idioma. entre más joven mejor

entre más experiencia presente el docente mejor

demostrada con certificaciones de haber realizado cursos de Ingles diferentes a los establecidos en los planes de estudio demostrado en los planes de estudio que acredite el docente. 